\title{
Número de anos para a estimação da média decendial de duração diária do brilho solar no Rio Grande do Sul
}

\author{
Number of years to estimate the ten-day average of daily sunshine duration in \\ Rio Grande do Sul State, Brazil
}

\author{
Alberto Cargnelutti Filho ${ }^{\mathrm{I}}$ Ronaldo Matzenauer ${ }^{\mathrm{II}}$ Bernadete Radin $^{\mathrm{II}}$ \\ Jaime Ricardo Tavares Maluf ${ }^{\text {II }}$
}

\section{RESUMO}

O objetivo deste trabalho foi determinar o tamanho de amostra (número de anos) para a estimação da média decendial de duração diária de brilho solar em 30 locais do Rio Grande do Sul. Com os dados de duração de brilho solar do período de 1960 a 2007, formaram-se 1.080 séries temporais (30 locais x 36 decêndios) de média decendial de duração diária de brilho solar. Testou-se a aleatoriedade e a normalidade dos dados, de cada série temporal, por meio dos testes de sequência (run test) e de Lilliefors, respectivamente. Para cada decêndio e local, foi determinado o tamanho de amostra por meio de reamostragem bootstrap, com reposição de 3.000 amostras. Vinte e cinco anos de observações são suficientes para a estimação da média decendial de duração diária de brilho solar, com amplitude do intervalo de confiança de bootstrap de 95\% igual a 2,00 horas dia ${ }^{-1}$.

Palavras-chave: tamanho de amostra, série temporal, reamostragem bootstrap, intervalo de confiança.

\section{ABSTRACT}

The objective of this research was to determine the sample size (number of years) to estimate the ten-day average of daily sunshine duration in 30 locations of the Rio Grande do Sul State, Brazil. With sunshine data duration of the period from 1960 to 2007, 1,080 time series (30 locations x 36 tendays) of ten-day of daily sunshine duration average were formed. The aleatory and normality, in each time series, was verified through the run test and Lilliefors test, respectively. For each ten-day and locality, it was determined the sample size using bootstrap resampling with replacement of 3,000 samples. Twenty-five years of data are enough to predict the ten-day average of daily sunshine duration, with amplitude of bootstrap confidence interval of 95\%, equal to 2.00 hour day ${ }^{-1}$.

Key words: sample size, time series, bootstrap resampling, confidence interval.

\section{INTRODUÇÃO}

O brilho solar, popularmente chamado de insolação, é representado pelas horas do dia em que o disco solar permanece visível à superfície terrestre, em local com horizonte desobstruído (VAREJÃO-SILVA, 2001). Esse elemento meteorológico é importante para o planejamento adequado de atividades agropecuárias. Interferências da duração do brilho solar sobre o desenvolvimento de culturas agrícolas têm sido discutidas em batata (BAPTISTA et al., 2006), girassol (MELLO et al., 2006), cana-de-açúcar (GOUVEA et al., 2009) e meloeiro (PEREIRA et al., 2010). Portanto, em um determinado período do ano (mês, quinzena, decêndio, semana, dia e hora) e local, a duração do brilho solar, para algumas aplicações, necessita ser estimada com precisão.

Para estudos de variabilidade e de mudança climática, a World Meteorological Organization (WMO, 2004) recomenda o uso de no mínimo 30 anos de observações. Diante disso, a estimação de parâmetros de elementos meteorológicos tem sido, comumente, realizada a partir de séries temporais de 30 anos de dados climáticos (normais climatológicas) independentemente da época do ano e do local.

O tamanho de amostra (número de anos) para a estimação da média mensal de duração diária do brilho solar em locais dos Estados do Rio Grande do Sul (CARGNELUTTI FILHO et al., 2010b) e de São Paulo

IDepartamento de Fitotecnia, Centro de Ciências Rurais (CCR), Universidade Federal de Santa Maria (UFSM), 97105-900, Santa Maria, RS, Brasil. E-mail: cargnelutti@pq.cnpq.br. Autor para correspondência.

IIFundação Estadual de Pesquisa Agropecuária (FEPAGRO), Porto Alegre, RS, Brasil. 
(MARTIN et al., 2008) tem sido investigado. De maneira geral, esses estudos revelaram que há variabilidade do tamanho de amostra no tempo e no espaço. Como consequência, o uso de 30 anos de observações, como preconizado pela World Meteorological Organization (WMO, 2004), de forma generalizada, não contempla a variabilidade dos dados de cada local e época do ano e gera estimativas de médias com precisões diferenciadas.

Os elementos meteorológicos podem ser definidos em escalas anual, mensal, quinzenal, decendial, semanal, diária e horária. De maneira geral, quanto menor a escala, a exemplo da horária, maior detalhamento pode ser realizado no planejamento de atividades agropecuárias. Portanto, é importante estimar os elementos meteorológicos, com precisão desejada, em escalas menores possíveis. Apesar dos estudos existentes sobre o dimensionamento do número de anos para a estimação da média mensal de duração diária do brilho solar (MARTIN et al., 2008; CARGNELUTTI FILHO et al., 2010b), não foram encontrados, na literatura, estudos dessa natureza, em escala decendial. Assim, o objetivo deste trabalho foi determinar o tamanho de amostra (número de anos) para a estimação da média decendial de duração diária de brilho solar em 30 locais do Rio Grande do Sul.

\section{MATERIAL E MÉTODOS}

Foram utilizados os dados de duração diária do brilho solar de 30 estações agrometeorológicas, oriundas de 30 locais do Rio Grande do Sul (Figura 1). Esses dados foram obtidos do Banco de Dados do Centro de Meteorologia Aplicada, da Fundação Estadual de Pesquisa Agropecuária (FEPAGRO/SCT-RS), e coletados no período de 1960 a 2007.

Em cada local e ano, a partir dos dados diários de duração do brilho solar, calculou-se a média decendial de duração do brilho solar, em horas dia $^{-1}$, de cada um dos 36 decêndios do ano. Assim, formaram-se 1.080 séries temporais de média decendial de duração diária do brilho solar (30 locais x 36 decêndios), com número diferenciado de anos de observações em cada série e definido em função da disponibilidade de dados meteorológicos.

Com a finalidade de identificar possíveis tendências de acréscimo ou decréscimo da média decendial de duração diária do brilho solar no período estudado, verificou-se a aleatoriedade dos dados em cada uma das 1.080 séries temporais, por meio do teste de sequência (run test) (SPIEGEL et al., 2004; SIEGEL \& CASTELLAN JÚNIOR, 2006). Foi aplicado um teste bilateral à hipótese $\mathrm{H}_{0}$ : a série é aleatória (sem tendência) versus a hipótese $\mathrm{H}_{1}$ : a série não é aleatória (com tendência). No teste, os dados de média decendial de duração diária do brilho solar foram utilizados em ordem cronológica, e o número de sequências foi calculado com base na média. Em seguida, foi verificada a normalidade dos dados de cada uma das 1.080 séries temporais por meio do teste de Lilliefors (CAMPOS, 1983), verificando-se as hipóteses $\mathrm{H}_{0}$ : os dados da série temporal se ajustam à distribuição normal versus a hipótese $\mathrm{H}_{1}$ : os dados da série temporal não se ajustam à distribuição normal.

A seguir, foram definidos 179 tamanhos de amostra para cada uma das 1.080 séries temporais. $\mathrm{O}$ tamanho de amostra inicial foi de dois anos e os demais foram obtidos com incrementos de um ano até atingir 180 anos. O valor 180 foi arbitrário e não foram simulados tamanhos maiores a esse, devido à dificuldade de se obter séries temporais com quantidades iguais ou superiores a 180 anos. Após, para cada tamanho de amostra simulado, de cada uma das 1.080 séries temporais, foram realizadas 3.000 simulações, por meio de reamostragem bootstrap, com reposição, o que totalizou 583.200.000 amostras simuladas (179 tamanhos simulados/série x 3.000 amostras simuladas/tamanho simulado x 1.080 séries). Para cada amostra simulada, foi estimada a média. Assim, para cada tamanho de amostra, de cada série temporal, foram obtidas 3.000 estimativas de bootstrap da média (FERREIRA, 2009) e determinados os valores mínimo, percentil 2,5\%, média, percentil 97,5\% e máximo. Depois, calculou-se a amplitude do intervalo de confiança de bootstrap de $95 \%$, pela diferença entre o percentil $97,5 \%$ e o percentil $2,5 \%$.

A seguir, determinou-se o tamanho de amostra (número de anos) para a estimação da média decendial de duração diária do brilho solar de cada uma das 1.080 séries temporais, com dois níveis de precisão. Para essa determinação, partiu-se do tamanho inicial simulado (dois anos), e considerou-se como tamanho de amostra, o número de anos a partir do qual a amplitude do intervalo de confiança de bootstrap de $95 \%$ foi menor ou igual a 1,00 hora dia- $^{-1}$ (maior precisão) e também menor ou igual a 2,00 horas dia $^{-1}$ (menor precisão). As análises estatísticas foram realizadas com o auxílio do programa R (R DEVELOPMENT CORE TEAM, 2011) e do aplicativo Office Excel.

\section{RESULTADOS E DISCUSSÃO}

Entre as 1.080 séries temporais de média decendial de duração diária do brilho solar, o teste de aleatoriedade (run test) evidenciou que 1.048 séries $(97,04 \%)$ foram aleatórias $(\mathrm{P}>0,05)$. Por outro lado, apenas 32 séries (2,96\%) foram não aleatórias $(\mathrm{P} \leq 0,05)$. Portanto, no período estudado, houve predomínio de 


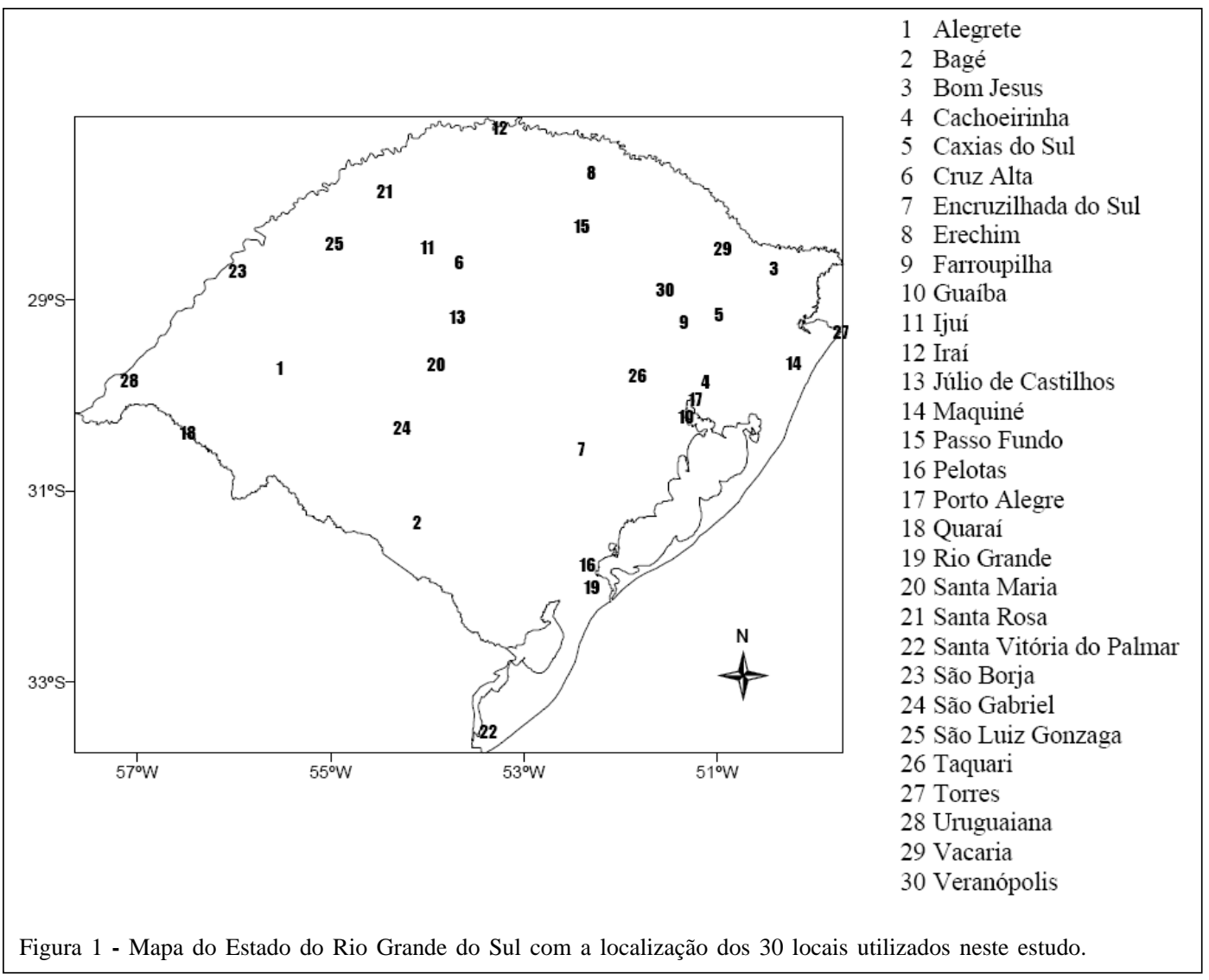

séries temporais aleatórias, o que significa, de maneira geral, que a média decendial de duração diária do brilho solar não apresentou tendência de acréscimo ou decréscimo. Percentuais semelhantes foram obtidos em relação à aleatoriedade de 312 séries de radiação solar global (BURIOL et al., 2006), 228 séries temporais de precipitação pluvial mensal (CARGNELUTTI FILHO et al., 2010a) e 360 séries temporais de média mensal de duração diária do brilho solar (CARGNELUTTI FILHO et al., 2010b). Para uma investigação adequada do tamanho de amostra, é importante que os dados amostrais sejam aleatórios (SIEGEL \& CASTELLAN JÚNIOR, 2006).

Os resultados do teste de Lilliefors revelaram que os dados de 990 séries temporais (91,67\%) de média decendial de duração diária do brilho solar se ajustaram à distribuição normal $(\mathrm{P}>0,05)$. A determinação do tamanho de amostra por meio de reamostragem bootstrap é uma alternativa adequada para essas 990 séries temporais com normalidade e para as demais 90 séries temporais (8,33\%), cujos dados não se ajustaram à distribuição normal $(\mathrm{P} \leq 0,05)$, pois essa metodologia independe da distribuição de probabilidade dos dados (FERREIRA, 2009). Então, diante dessas considerações, em relação à aleatoriedade e à normalidade, pode-se inferir que os dados dessas 1.080 séries temporais de média decendial de duração diária do brilho solar conferem credibilidade ao estudo do tamanho de amostra, com base na metodologia de reamostragem bootstrap.

Nos dois casos extremos (Figuras 2-A e 2-B), e nas demais 1.078 series temporais intermediárias, a amplitude do intervalo de confiança de bootstrap de 95\% (percentil 97,5\% - percentil 2,5\%) diminuiu, gradativamente, com o acréscimo do tamanho de amostra (número de anos). Isso significa, como esperado, maior precisão da estimativa da média decendial de duração diária do brilho solar com mais anos de observações. Visualmente, percebe-se que o decréscimo da amplitude (aumento da precisão) é mais evidente até cerca de 30 anos de observações. Depois disso, o acréscimo do número de anos fornece ganhos inexpressivos na precisão das estimativas. Esses resultados parecem evidenciar que seriam suficientes em torno de 30 anos de observações para a estimação da média decendial de duração diária do brilho solar.

O tamanho de amostra (número de anos) para a estimação da média (m) decendial de duração diária do brilho solar, em 30 locais do Rio Grande do 


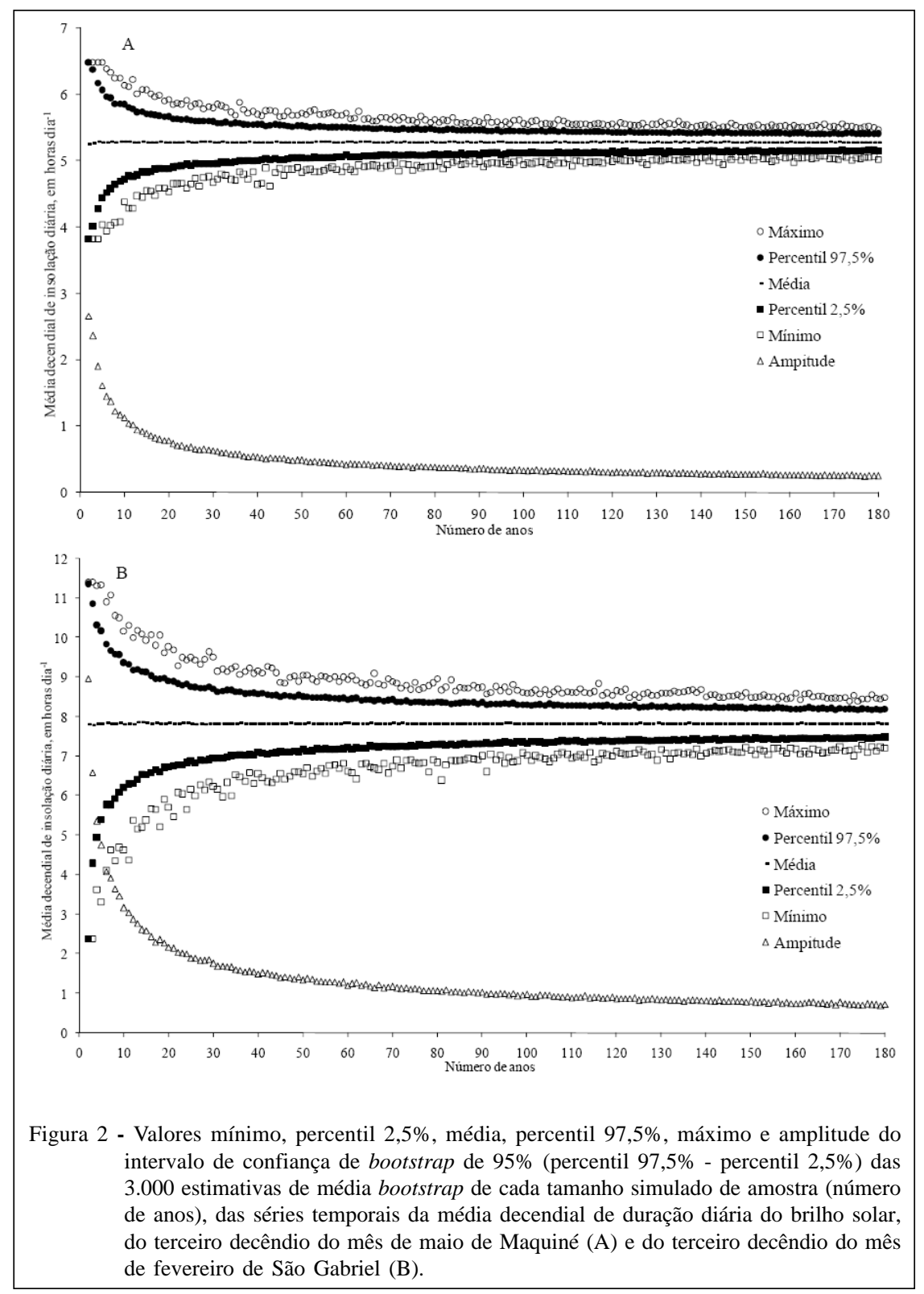

Sul, para uma amplitude do intervalo de confiança de bootstrap de 95\% igual a 1,00 hora $\mathrm{dia}^{-1}$, oscilou entre 13 anos no terceiro decêndio do mês de maio, em Maquiné (Figura 2-A), e 91 anos no terceiro decêndio do mês de fevereiro, em São Gabriel (Figura 2-B) e a média das 1.080 séries temporais foi de 46 anos. Portanto, pode-se inferir que, na prática, a obtenção de estimativas, em escala decendial, com essa precisão, é difícil, pelo elevado número de observações (anos) necessárias.

Com a amplitude do intervalo de confiança de bootstrap de $95 \%$ igual a 2,00 horas dia $^{-1}$ (menor precisão), o tamanho de amostra oscilou entre 4 anos no terceiro decêndio do mês de maio, em Maquiné (Tabela 1), e 25 anos no terceiro decêndio do mês de fevereiro, em São Gabriel (Tabela 2). Então, na prática, tomando-se como referência o maior tamanho de amostra, pode-se inferir que 25 anos de observações são suficientes para a estimação da média decendial de duração diária do brilho solar, com intervalo de confiança de bootstrap de 95\% de 2,00 horas dia $^{-1}$.

Partindo do referencial de 30 anos (normal climatológica), a amplitude do intervalo de confiança de bootstrap de $95 \%$ oscilou entre 0,63 horas dia $^{-1}$ no terceiro decêndio do mês de maio, em Maquiné (Figura 2-A), e

Ciência Rural, v.42, n.3, mar, 2012. 
1,76 horas dia ${ }^{-1}$ no terceiro decêndio do mês de fevereiro, em São Gabriel (Figura 2-B), e a média das 1.080 séries temporais foi de 1,23 horas dia $^{-1}$. Então, pode-se inferir que há variabilidade nas precisões das estimativas da média decendial de duração diária do brilho solar, com base em um número fixo de anos, entre os decêndios e os locais. Tamanhos de amostra com variabilidade espacial e temporal também foram encontrados por CARGNELUTTI FILHO et al. (2010b) e MARTIN et al.
(2008) em estudos para a estimação da média mensal de duração diária do brilho solar.

\section{CONCLUSÃO}

Vinte e cinco anos de observações são suficientes para a estimação da média decendial de duração diária do brilho solar, com amplitude do intervalo de confiança de bootstrap de 95\% igual a

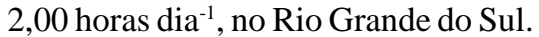

Tabela 1 - Tamanho de amostra (número de anos) para a estimação da média decendial de duração diária do brilho solar em 15 locais do Estado do Rio Grande do Sul, com amplitude do intervalo de confiança de bootstrap de 95\% de 2,00 horas dia ${ }^{-1}$.

\begin{tabular}{|c|c|c|c|c|c|c|c|c|c|c|c|c|c|c|c|c|}
\hline$\stackrel{\mathscr{d}}{\Sigma}^{\infty}$ & 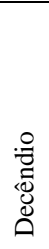 & 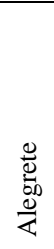 & 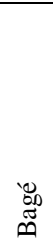 & 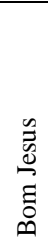 & 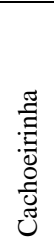 & 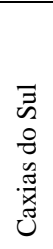 & 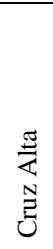 & 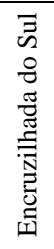 & 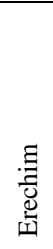 & 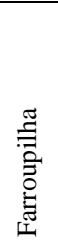 & 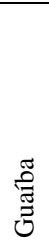 & $\Xi$ & “7ే & 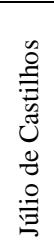 & 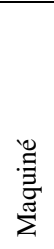 & 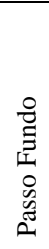 \\
\hline Janeiro & 1 & 17 & 13 & 10 & 14 & 13 & 12 & 17 & 18 & 15 & 13 & 17 & 14 & 14 & 13 & 13 \\
\hline Janeiro & 2 & 13 & 15 & 20 & 20 & 18 & 19 & 19 & 18 & 19 & 20 & 17 & 15 & 21 & 18 & 18 \\
\hline Janeiro & 3 & 14 & 15 & 13 & 11 & 13 & 14 & 16 & 13 & 11 & 10 & 11 & 12 & 13 & 17 & 12 \\
\hline Fevereiro & 1 & 12 & 11 & 8 & 15 & 12 & 13 & 14 & 13 & 12 & 16 & 11 & 13 & 11 & 10 & 12 \\
\hline Fevereiro & 2 & 14 & 12 & 14 & 11 & 12 & 11 & 14 & 17 & 12 & 13 & 13 & 14 & 13 & 14 & 13 \\
\hline Fevereiro & 3 & 21 & 17 & 17 & 18 & 16 & 19 & 22 & 18 & 19 & 22 & 18 & 18 & 20 & 16 & 20 \\
\hline Março & 1 & 11 & 9 & 7 & 9 & 11 & 7 & 11 & 10 & 9 & 6 & 10 & 8 & 10 & 9 & 9 \\
\hline Março & 2 & 10 & 8 & 12 & 16 & 16 & 15 & 13 & 14 & 17 & 20 & 14 & 15 & 11 & 15 & 13 \\
\hline Março & 3 & 12 & 11 & 12 & 9 & 12 & 13 & 13 & 16 & 8 & 7 & 12 & 13 & 11 & 7 & 15 \\
\hline Abril & 1 & 13 & 11 & 12 & 13 & 12 & 15 & 14 & 10 & 12 & 16 & 11 & 12 & 12 & 11 & 12 \\
\hline Abril & 2 & 13 & 16 & 14 & 14 & 18 & 18 & 16 & 15 & 19 & 15 & 17 & 13 & 13 & 11 & 15 \\
\hline Abril & 3 & 11 & 11 & 16 & 14 & 16 & 14 & 17 & 14 & 16 & 11 & 16 & 16 & 15 & 10 & 14 \\
\hline Maio & 1 & 11 & 7 & 13 & 11 & 16 & 13 & 12 & 15 & 14 & 14 & 13 & 12 & 14 & 15 & 15 \\
\hline Maio & 2 & 10 & 7 & 8 & 6 & 12 & 10 & 12 & 12 & 11 & 7 & 13 & 10 & 9 & 6 & 12 \\
\hline Maio & 3 & 11 & 9 & 12 & 7 & 11 & 12 & 11 & 12 & 9 & 9 & 11 & 9 & 11 & 4 & 9 \\
\hline Junho & 1 & 11 & 10 & 10 & 7 & 12 & 14 & 12 & 17 & 10 & 10 & 14 & 11 & 14 & 7 & 15 \\
\hline Junho & 2 & 13 & 12 & 12 & 9 & 14 & 11 & 12 & 13 & 10 & 11 & 12 & 10 & 12 & 8 & 13 \\
\hline Junho & 3 & 9 & 10 & 12 & 8 & 12 & 10 & 9 & 12 & 9 & 7 & 11 & 10 & 10 & 9 & 10 \\
\hline Julho & 1 & 16 & 9 & 11 & 8 & 12 & 15 & 12 & 14 & 10 & 12 & 14 & 10 & 13 & 8 & 13 \\
\hline Julho & 2 & 10 & 11 & 14 & 8 & 9 & 10 & 10 & 12 & 11 & 10 & 10 & 9 & 9 & 13 & 10 \\
\hline Julho & 3 & 11 & 11 & 12 & 10 & 10 & 14 & 12 & 12 & 11 & 13 & 11 & 8 & 13 & 8 & 10 \\
\hline Agosto & 1 & 10 & 11 & 14 & 15 & 12 & 13 & 14 & 12 & 14 & 13 & 13 & 10 & 12 & 9 & 12 \\
\hline Agosto & 2 & 12 & 11 & 15 & 16 & 19 & 19 & 16 & 17 & 22 & 19 & 16 & 14 & 15 & 15 & 16 \\
\hline Agosto & 3 & 11 & 12 & 9 & 9 & 9 & 12 & 10 & 12 & 10 & 10 & 10 & 9 & 10 & 6 & 10 \\
\hline Setembro & 1 & 14 & 14 & 12 & 18 & 16 & 12 & 18 & 10 & 14 & 14 & 17 & 14 & 18 & 9 & 15 \\
\hline Setembro & 2 & 13 & 11 & 13 & 12 & 16 & 16 & 10 & 17 & 12 & 10 & 11 & 15 & 10 & 9 & 14 \\
\hline Setembro & 3 & 14 & 15 & 13 & 12 & 16 & 13 & 16 & 15 & 15 & 7 & 14 & 16 & 12 & 11 & 17 \\
\hline Outubro & 1 & 12 & 14 & 8 & 9 & 10 & 10 & 12 & 11 & 9 & 12 & 11 & 14 & 12 & 6 & 10 \\
\hline Outubro & 2 & 9 & 9 & 13 & 11 & 14 & 14 & 20 & 14 & 15 & 10 & 13 & 13 & 16 & 18 & 17 \\
\hline Outubro & 3 & 11 & 10 & 12 & 10 & 12 & 10 & 12 & 13 & 12 & 11 & 9 & 11 & 12 & 11 & 10 \\
\hline Novembro & 1 & 18 & 16 & 13 & 16 & 13 & 15 & 19 & 14 & 15 & 16 & 14 & 20 & 12 & 23 & 12 \\
\hline Novembro & 2 & 10 & 10 & 13 & 10 & 11 & 9 & 14 & 14 & 12 & 12 & 10 & 13 & 9 & 12 & 9 \\
\hline Novembro & 3 & 12 & 10 & 9 & 9 & 16 & 15 & 14 & 13 & 12 & 18 & 12 & 14 & 13 & 10 & 13 \\
\hline Dezembro & 1 & 11 & 13 & 11 & 12 & 9 & 13 & 17 & 16 & 12 & 14 & 12 & 14 & 13 & 9 & 13 \\
\hline Dezembro & 2 & 8 & 9 & 11 & 8 & 10 & 9 & 12 & 13 & 11 & 6 & 11 & 11 & 10 & 8 & 11 \\
\hline Dezembro & 3 & 12 & 10 & 12 & 12 & 16 & 10 & 17 & 15 & 15 & 15 & 11 & 9 & 10 & 17 & 9 \\
\hline
\end{tabular}


Tabela 2 - Tamanho de amostra (número de anos) para a estimação da média decendial de duração diária do brilho solar em 15 locais do Estado do Rio Grande do Sul, com amplitude do intervalo de confiança de bootstrap de 95\% de 2,00 horas dia ${ }^{-1}$.

\begin{tabular}{|c|c|c|c|c|c|c|c|c|c|c|c|c|c|c|c|c|}
\hline$\sum_{\Sigma}^{\mathscr{e}}$ & 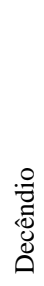 & $\frac{\tilde{J}}{\frac{\pi}{0}}$ & 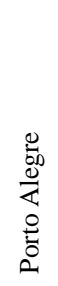 & 莡 & 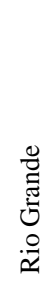 & 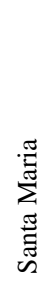 & 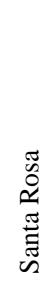 & 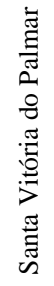 & 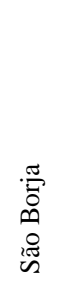 & 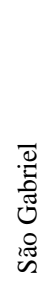 & 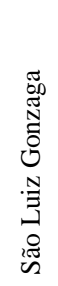 & 䔍 & 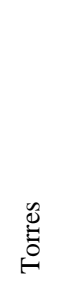 & 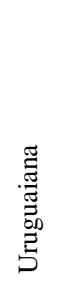 & $\begin{array}{l}\stackrel{\pi}{\tilde{J}} \\
\underset{\pi}{J}\end{array}$ & $\begin{array}{l}\stackrel{n}{\circ} \\
\stackrel{0}{\circ} \\
\stackrel{0}{0} \\
>\end{array}$ \\
\hline Janeiro & 1 & 12 & 12 & 17 & 12 & 18 & 10 & 12 & 16 & 15 & 13 & 12 & 12 & 11 & 20 & 13 \\
\hline Janeiro & 2 & 19 & 19 & 16 & 18 & 23 & 21 & 12 & 17 & 19 & 20 & 23 & 17 & 16 & 23 & 18 \\
\hline Janeiro & 3 & 13 & 13 & 13 & 14 & 17 & 13 & 14 & 11 & 16 & 16 & 11 & 13 & 19 & 11 & 11 \\
\hline Fevereiro & 1 & 12 & 12 & 13 & 11 & 15 & 12 & 11 & 15 & 12 & 14 & 12 & 12 & 16 & 11 & 11 \\
\hline Fevereiro & 2 & 11 & 11 & 14 & 13 & 14 & 12 & 12 & 14 & 17 & 14 & 15 & 17 & 16 & 15 & 13 \\
\hline Fevereiro & 3 & 23 & 18 & 19 & 20 & 24 & 14 & 23 & 14 & 25 & 18 & 20 & 18 & 21 & 17 & 21 \\
\hline Março & 1 & 8 & 9 & 14 & 15 & 12 & 10 & 8 & 10 & 12 & 11 & 6 & 7 & 14 & 9 & 6 \\
\hline Março & 2 & 10 & 13 & 10 & 12 & 12 & 10 & 9 & 12 & 14 & 13 & 15 & 15 & 9 & 13 & 17 \\
\hline Março & 3 & 10 & 9 & 10 & 11 & 12 & 10 & 9 & 8 & 12 & 12 & 9 & 7 & 14 & 10 & 10 \\
\hline Abril & 1 & 13 & 11 & 11 & 9 & 15 & 14 & 8 & 14 & 16 & 14 & 15 & 12 & 17 & 10 & 12 \\
\hline Abril & 2 & 16 & 14 & 11 & 17 & 16 & 18 & 9 & 13 & 20 & 17 & 15 & 14 & 17 & 17 & 15 \\
\hline Abril & 3 & 14 & 15 & 11 & 13 & 12 & 15 & 9 & 13 & 17 & 16 & 15 & 11 & 17 & 12 & 15 \\
\hline Maio & 1 & 8 & 12 & 9 & 9 & 15 & 13 & 8 & 11 & 17 & 13 & 17 & 14 & 12 & 15 & 16 \\
\hline Maio & 2 & 10 & 9 & 9 & 12 & 12 & 13 & 9 & 10 & 10 & 11 & 12 & 11 & 12 & 10 & 12 \\
\hline Maio & 3 & 11 & 9 & 11 & 9 & 12 & 14 & 10 & 10 & 12 & 14 & 11 & 10 & 11 & 9 & 9 \\
\hline Junho & 1 & 12 & 8 & 14 & 11 & 13 & 8 & 12 & 13 & 16 & 14 & 12 & 11 & 11 & 11 & 13 \\
\hline Junho & 2 & 13 & 9 & 11 & 10 & 13 & 16 & 13 & 10 & 9 & 16 & 11 & 14 & 16 & 10 & 10 \\
\hline Junho & 3 & 9 & 8 & 11 & 10 & 10 & 10 & 11 & 14 & 10 & 11 & 9 & 9 & 12 & 10 & 9 \\
\hline Julho & 1 & 11 & 10 & 12 & 11 & 12 & 10 & 9 & 13 & 12 & 13 & 12 & 11 & 13 & 15 & 10 \\
\hline Julho & 2 & 9 & 8 & 10 & 12 & 11 & 8 & 8 & 9 & 14 & 9 & 10 & 9 & 12 & 10 & 11 \\
\hline Julho & 3 & 11 & 9 & 14 & 11 & 13 & 11 & 10 & 10 & 12 & 13 & 12 & 11 & 11 & 9 & 10 \\
\hline Agosto & 1 & 15 & 12 & 12 & 12 & 14 & 13 & 11 & 11 & 15 & 12 & 13 & 11 & 14 & 13 & 14 \\
\hline Agosto & 2 & 14 & 15 & 10 & 12 & 16 & 12 & 11 & 13 & 16 & 15 & 20 & 14 & 11 & 17 & 18 \\
\hline Agosto & 3 & 13 & 9 & 10 & 10 & 12 & 13 & 9 & 7 & 11 & 9 & 12 & 11 & 11 & 9 & 11 \\
\hline Setembro & 1 & 13 & 19 & 14 & 14 & 19 & 13 & 9 & 14 & 16 & 17 & 22 & 12 & 17 & 12 & 14 \\
\hline Setembro & 2 & 12 & 13 & 10 & 11 & 13 & 13 & 9 & 10 & 12 & 12 & 12 & 12 & 12 & 15 & 11 \\
\hline Setembro & 3 & 14 & 13 & 14 & 14 & 15 & 15 & 12 & 14 & 18 & 15 & 14 & 14 & 15 & 12 & 13 \\
\hline Outubro & 1 & 12 & 10 & 14 & 14 & 14 & 9 & 11 & 11 & 16 & 11 & 12 & 9 & 15 & 11 & 10 \\
\hline Outubro & 2 & 9 & 13 & 10 & 12 & 13 & 16 & 10 & 12 & 14 & 14 & 10 & 19 & 15 & 18 & 15 \\
\hline Outubro & 3 & 10 & 12 & 9 & 10 & 11 & 13 & 8 & 11 & 12 & 13 & 13 & 11 & 11 & 17 & 13 \\
\hline Novembro & 1 & 15 & 14 & 16 & 18 & 14 & 14 & 16 & 16 & 17 & 15 & 16 & 13 & 18 & 10 & 14 \\
\hline Novembro & 2 & 12 & 9 & 9 & 15 & 13 & 7 & 13 & 10 & 13 & 10 & 10 & 16 & 12 & 14 & 10 \\
\hline Novembro & 3 & 11 & 10 & 8 & 11 & 13 & 14 & 9 & 13 & 15 & 13 & 12 & 14 & 13 & 12 & 11 \\
\hline Dezembro & 1 & 14 & 11 & 12 & 13 & 18 & 12 & 12 & 9 & 19 & 14 & 13 & 10 & 12 & 13 & 13 \\
\hline Dezembro & 2 & 9 & 11 & 9 & 11 & 15 & 10 & 10 & 8 & 10 & 13 & 9 & 10 & 11 & 8 & 10 \\
\hline Dezembro & 3 & 13 & 13 & 12 & 11 & 19 & 9 & 11 & 11 & 11 & 14 & 14 & 19 & 11 & 12 & 11 \\
\hline
\end{tabular}

\section{AGRADECIMENTOS}

Aos pesquisadores, aos técnicos, aos observadores meteorológicos, aos estagiários e as demais pessoas que, de alguma forma, contribuíram para a realização deste trabalho; ao Conselho Nacional de Desenvolvimento Científico e Tecnológico (CNPq), pela concessão de bolsa de produtividade em pesquisa para Alberto Cargnelutti Filho.

\section{REFERÊNCIAS}

BAPTISTA, M.J. et al. Efeito da solarização e biofumigação, durante o outono, na incidência de murcha-bacteriana e produtividade da batata. Horticultura Brasileira, v.24, p.99102, 2006. Disponível em: <http://www.scielo.br/ scielo.php? script =sci arttext\&pid=S0102$05362006000100020 \& \operatorname{lng}=$ pt $\& \mathrm{nrm}=$ iso $>$. Acesso em: 10 maio, 2011. doi: 10.1590/S0102-05362006000100020. 
BURIOL, G.A. et al. Homogeneidade da radiação solar global medida nas estações agrometeorológicas da Fundação Estadual de Pesquisa Agropecuária do Estado do Rio Grande do Sul, Brasil. Pesquisa Agropecuária Gaúcha, v.12, p.65-72, 2006.

CAMPOS, H. de Estatística experimental não-paramétrica. 4.ed. Piracicaba: Departamento de Matemática e Estatística ESALQ, 1983. 349p.

CARGNELUTTI FILHO, A. et al. Dimensionamento da amostra para a estimação da média de precipitação pluvial mensal em diferentes locais do Estado do Rio Grande do Sul. Ciência Rural, v.40, p.12-19, 2010a. Disponível em: <http:// www.scielo.br/scielo.php?script=sci_arttext\&pid=S0103$84782010000100003 \& \operatorname{lng}=p t \& n r m=i s o>$. Acesso em: 10 maio, 2011. doi: 10.1590/S0103-84782009005000226.

CARGNELUTTI FILHO, A. et al. Tamanho de amostra para a estimação da média mensal de insolação diária em diferentes locais do estado do Rio Grande do Sul. Ciência Rural, v.40, p.1509-1515, 2010b. Disponível em: <http://www.scielo.br/ s c i e l o.ph p ? s c ri p t = s ci_art t ext \& pid = S 0103 $84782010000700005 \& \operatorname{lng}=p t \& n r m=i s o>$. Acesso em: 10 maio, 2011. doi: 10.1590/S0103-84782010005000112.

FERREIRA, D.F. Estatística básica. 2.ed. Lavras: UFLA, 2009. 664p.

GOUVEA, J.R.F. et al. Climate changes and technological advances: impacts on sugarcane productivity in tropical southern Brazil. Scientia Agricola, v.66, p.593-605, 2009. Disponível em: <http:/ /www.scielo.br/scielo.php?script=sci_arttext \&pid=S010390162009000500003\&lng=pt\&nrm=iso $>$. Acesso em: 10 maio, 2011. doi: 10.1590/S0103-90162009000500003.

MARTIN, T.N. et al. Regiões homogêneas e tamanho de amostra para atributos do clima no Estado de São Paulo, Brasil. Ciência Rural, v.38, p.690-697, 2008. Disponível em: <http:/ /www.scielo.br/scielo.php?script=sci_arttext\&pid=S0103-
$84782008000300015 \& \operatorname{lng}=$ pt $\&$ nrm $=$ iso $>$. Acesso em: 10 maio, 2011. doi: 10.1590/S0103-84782008000300015.

MELLO, R. et al. Características fenológicas, produtivas e qualitativas de híbridos de girassol em diferentes épocas de semeadura para produção de silagem. Revista Brasileira de Zootecnia, v.35, p.672-682, 2006. Disponível em: <http:// www.scielo.br/scielo.php?script=sci_arttext\&pid=S1516 $35982006000300007 \& \operatorname{lng}=p t \& n r m=i s o>$. Acesso em: 10 maio, 2011. doi: 10.1590/S1516-35982006000300007.

PEREIRA, F.H.F. et al. Produção e qualidade de frutos de melões amarelo e charentais cultivados em ambientes sombreados. Revista Brasileira de Engenharia Agrícola e Ambiental, v.14, p.944-950, 2010. Disponível em: <http://www.scielo.br/ s c i e lo.ph p ? s cript = sci_art text \& pid = S 1415 43662010000900006\&lng=pt\&nrm=iso $>$. Acesso em: 10 maio, 2011. doi: 10.1590/S1415-43662010000900006.

R DEVELOPMENT CORE TEAM. R: A language and environment for statistical computing. Vienna: $R$ Foundation for Statistical Computing, 2011. Disponível em: http://www.R-project.org. Acesso em: 10 maio, 2011.

SIEGEL, S.; CASTELLAN JÚNIOR, N.J. Estatística nãoparamétrica para ciências do comportamento. 2.ed. Porto Alegre: Artmed, 2006. 448p.

SPIEGEL, R.A. et al. Probabilidade e estatística. 2.ed Porto Alegre: Bookman, 2004. 398p.

VAREJÃO-SILVA, M.A. Meteorologia e Climatologia. Ministério da Agricultura e do Abastecimento. 2.ed. Brasília: INMET, 2001. 532p.

WORLD METEOROLOGICAL ORGANIZATION (WMO). Implementation Plan for the Global Observing System for Climate in Support of the UNFCCC, 2004. 29p. (WMO/TD n. 1244). Disponível em: http://www.wmo.int/pages/ prog/gcos/Publications/gcos-92_GIP_ES.pdf. Acesso em: 26 jan. 2011. 